hep-ph/0009211

\title{
Effects of the scale-dependent vacuum expectation values in the renormalisation group analysis of neutrino masses
}

\author{
N.Nimai Singh円 \\ Department of Physics and Astronomy, University of Southampton, Southampton, \\ SO17 1BJ, U.K.
}

\begin{abstract}
The contribution of scale-dependent vacuum expectation values (VEVs) of Higgs scalars, which gives significant effects in the evolution of fundamental fermion masses in the Minimal Supersymmetric Standard Model (MSSM), is now considered in the derivation of the one-loop analytic expression for the evolution of the left-handed Majorana neutrino masses with energies. The inclusion of such effect of the running VEV increases the stability of the neutrino masses under quantum corrections even for low values of $\tan \beta \geq 1.42$ at the scale $\mu=10^{12} \mathrm{GeV}$, and leads to a mild decrease of neutrino masses with higher energies. Such trend is common to that of other fundamental fermion masses.
\end{abstract}

${ }^{1}$ Permanent Address: Department of Physics, Gauhati University, Guwahati -781014, India 
In recent years a large number of theoretical papers were devoted to building models for generating small neutrino masses and lepton mixings within or outside the framework of the Grand Unified Theories (GUTs) with extended $U(1)$ group[1]. Both analytic and numerical studies[2,3,4] have been carried out for checking the stability of the textures of neutrino mass matrix and lepton mixing matrix under quantum radiative corrections[5]. There are basically two approaches: the top-down approach[2] which predicts the neutrino masses and mixings in terms of GUT-parameters, and the bottom-up approach [6] which predicts the running parameters at higher scales in terms of experimentally determined values at low energies. In the top-down programme, one usually starts with the running of a set of the RGEs for Yukawa matrices and gauge couplings in the MSSM (or SM), with three right-handed heavy neutrinos, taking into account the effects of the heavy neutrino mass thresholds, from GUT scale down to the lightest right-handed neutrino mass scale $\left(M_{R 1}\right)$. This fixes the lefthanded Majorana neutrino mass matrix $m_{L L}\left(M_{R 1}\right)$ through the see-saw mechanism[7] at this scale,

$$
m_{L L}\left(M_{R 1}\right)=v_{u}^{2} Y_{\nu}\left(M_{R 1}\right) M_{R R}^{-1} Y_{\nu}^{T}\left(M_{R 1}\right)
$$

Below this scale $M_{R 1}$ the right-handed neutrinos decouple from the theory, and the neutrino mass matrix in Eq.(1) is taken as[2]

$$
m_{L L}\left(M_{R 1}\right)=v_{u}^{2} \kappa\left(M_{R 1}\right)
$$

where $\kappa$ is the coefficient of the dimension 5 neutrino mass operator. In the energy range from $M_{R 1}$ down to low energy at $m_{t}$, the running of the coefficient $\kappa$ in the diagonal charged lepton basis, fixes the neutrino mass matrix at scale $m_{t}$,

$$
m_{L L}\left(m_{t}\right)=v_{u}^{2} \kappa\left(m_{t}\right)
$$

In the above discussion only the scale-dependence of $\kappa$ is considered, and not the running of the vacuum expectation value(VEV), $v_{u}$ in Eqs.(1)-(3). This led to the increase of neutrino mass eigenvalues with energy scales, giving significant effect for low 
$\tan \beta$ values. As it is strongly $\tan \beta$ dependent, this efect may lead to the instability of the neutrino masses under quantum radiative corrections I $^{2}$. For higher values of $\tan \beta$ the stability is again improved. Such increasing trend of neutrino mass eigenvalues with the increase in energies, is opposite to that of the general trend shown by other fundamental fermions (charged leptons and quarks) $[8,9]$. The effects of the contributions of the scale-dependent vacuum expectation values(VEVs) of Higgs scalars in the one-loop analytic expressions, in the evolution of quarks and charged leptons masses at higher energies in the MSSM, had been studied in Ref.(8), and this effect is quite significant.

In this paper we study the stability of the magnitudes of neutrino masses at low $\tan \beta$ and their running behaviour at different energies, by considering the scaledependence[10] of the vacuum expectation value (VEV), $v_{u}$, along with that of $\kappa$. The expression in Eq.(3) is now modified as

$$
m_{L L}(t)=v_{u}^{2}(t) \kappa(t)
$$

where $v_{u}\left(t_{0}\right)=v_{0} \sin \beta, v_{0}=174 \mathrm{GeV}, t=\ln \mu, t_{0}=\ln m_{t}$. The above equation(4) can be written as

$$
\frac{d \ln m_{L L}(t)}{d t}=\frac{d \ln \kappa(t)}{d t}+2 \frac{d \ln v_{u}(t)}{d t}
$$

where the second term on the right-hand side of the above equation is the contribution from the running of the VEV. The RGEs for $v_{u}[8,10]$ and $\kappa[2,5]$ in the diagonal charged lepton basis, for one-loop order in MSSM, in the energy range $t \geq t_{0}$, are given by

$$
\frac{d \ln v_{u}}{d t}=\frac{1}{16 \pi^{2}}\left[\frac{3}{20} g_{1}^{2}+\frac{3}{4} g_{2}^{2}-3 h_{t}^{2}\right]
$$

and,

$$
\frac{d \ln \kappa}{d t}=-\frac{1}{16 \pi^{2}}\left[\frac{6}{5} g_{1}^{2}+6 g_{2}^{2}-6 h_{t}^{2}-\delta_{i 3} h_{\tau}^{2}-\delta_{3 j} h_{\tau}^{2}\right]
$$

\footnotetext{
${ }^{2}$ In Refs. [3,4] the stability condition is decided by the change in texture of neutrino mass matrix only. Here we emphasise that changing pattern of the overall magnitudes of neutrino mass eigenvalues at different energies, may also cause instability.
} 
respectively. Substituition of Eqs.(6,7) in Eq.(5) gives

$$
\frac{d \ln m_{L L}}{d t}=\frac{1}{16 \pi^{2}}\left[-\frac{9}{10} g_{1}^{2}-\frac{9}{2} g_{2}^{2}+\delta_{i 3} h_{\tau}^{2}+\delta_{3 j} h_{\tau}^{2}\right]
$$

Upon integration from low scale $t_{0}=\ln m_{t}$ to high scale $t_{R 1}=\ln M_{R 1}$ where $t_{R 1} \geq t_{0}$, we get the correct expression for the neutrino mass matrix at $t_{0}$,

$$
\begin{gathered}
\frac{\left(m_{L L}\left(t_{0}\right)\right)_{i j}}{\left(m_{L L}\left(t_{R 1}\right)\right)_{i j}}=e^{\left(\frac{9}{10} I_{g 1}+\frac{9}{2} I_{g 2}\right)} e^{-I_{\tau}\left(\delta_{i 3}+\delta_{3 j}\right)}, \\
I_{f}=\frac{1}{16 \pi^{2}} \int_{\ln m_{t}}^{\ln M_{R 1}} h_{f}^{2}(t) d t, \\
I_{g_{i}}=\frac{1}{16 \pi^{2}} \int_{\ln m_{t}}^{\ln M_{R 1}} g_{i}^{2}(t) d t \simeq \ln \left(\frac{g_{i}\left(t_{R 1}\right)}{g_{i}\left(t_{0}\right)}\right)^{\left(1 / b_{i}\right)}
\end{gathered}
$$

where $f=t, \tau ; i=1,2,3$, and $b_{i}=(33 / 5,1,-3)$ for MSSM. The correct expression in Eq.(9) will certainly affect the earlier numerical results obtained without taking the effect of the running $\mathrm{VEV}[2]$ at scale $M_{R 1}$.

For simplicity we now follow the analysis of the RGEs for neutrino mass eigenvalues[4]. With the inclusion of such scale-dependence of VEV in Eq.(6), the RGEs for the mass eigenvalues given in Ref.[4] in the diagonal charged lepton basis, is now modified as,

$$
\frac{d \ln m_{\nu a}}{d t}=\frac{1}{16 \pi^{2}} \sum_{b=e, \mu, \tau}\left[-\frac{9}{10} g_{1}^{2}-\frac{9}{2} g_{2}^{2}+2 h_{b}^{2} V_{b a}^{2}\right]
$$

where $a=1,2,3$, and $V_{b a}$ is the MNS mixing matrix element. The correct expression for the neutrino mass ratio at different energy scales, is also obtained by integrating Eq. (12) as

$$
R_{a}\left(t_{R 1}\right)=\frac{m_{\nu a}\left(t_{R 1}\right)}{m_{\nu a}\left(t_{0}\right)} \approx e^{-\left(\frac{9}{10} I_{g 1}+\frac{9}{2} I_{g 2}\right)} e^{2 V_{\tau a}^{2} I_{\tau}}
$$

In getting Eq.(13) we have neglected very small effects due to $I_{\mu, e}$ compared to $I_{\tau}$, and also assumed $V_{\tau a}$ does not change much in the integration range For a typical value of the element of MNS mixing matrix $V_{\tau 3} \simeq \frac{1}{\sqrt{2}}$, we can get the condition

\footnotetext{
${ }^{3}$ Such approximation can be justified for the calculation of the mass eigenvalues and their ratios as the second exponential term in Eq.(13) gives almost 1 for low values of $I_{\tau}$.
} 
$m_{\nu 3}\left(t_{0}\right)>m_{\nu 3}\left(t_{R 1}\right)$ following Eq.(13), which shows a mild increase in neutrino masses with the decrease in energies, even for small $\tan \beta \geq 1.42$. This is due to the fact that the ratio $R_{3}\left(t_{R 1}\right)$ is now independent of $e^{6 I_{t}}$ in the first exponential factor in Eq.(13). The same is true in Eq.(9). In fact the contribution of the running VEV effectively makes the replacement in the exponential factor:

$$
e^{-\left(\frac{6}{5} I_{g 1}+6 I_{g 2}-6 I_{t}\right)} \rightarrow e^{-\left(\frac{9}{10} I_{g 1}+\frac{9}{2} I_{g 2}\right)}
$$

in Eqs. $(9,13)$.

We now study the effect of the running VEV in the evolution of squared neutrino mass difference, $\triangle m_{i j}^{2}=\left|m_{\nu i}^{2}-m_{\nu j}^{2}\right|$ with energies. By taking square on both sides of Eq.(13), and considering for two mass eigenvalues $a=i, j$, we get approximately,

$$
\triangle m_{i j}^{2}\left(t_{R 1}\right) \approx \triangle m_{i j}^{2}\left(t_{0}\right) e^{-2\left(\frac{9}{10} I_{g 1}+\frac{9}{2} I_{g 2}\right)} e^{4 V_{\tau i}^{2} I_{\tau}}
$$

where we assume that the small difference between $V_{\tau i}$ and $V_{\tau j}$ for $i, j=1,2,3$, does not alter much the last exponential term which can be approximately taken as $e^{4 V_{\tau i}^{2} I_{\tau}} \simeq e^{4 V_{\tau j}^{2} I_{\tau}} \approx 1$ for the low values of $I_{\tau}$. This amounts to neglecting small changes in the texture of neutrino mass matrix which would be relevant for the evolution of mixing angles. The evolution of $\Delta m_{i j}^{2}\left(t_{R 1}\right)$ is now stable with the effects of running VEV for both low and high values of $\tan \beta$, otherwise it would have been more strongly $\tan \beta$-dependent with $e^{12 I_{t}}$ in the exponential factor in the case where the effect of running VEV is not included, causing more instability at low $\tan \beta$ values.

The running of the ratio of two neutrino mass eigenvalues, $R_{23}=m_{\nu 2} / m_{\nu 3}$ (and hence the running of $R R_{23}$ ) is independent of the effect of running VEV, so that the ratio of ratios is,

$$
R R_{23}\left(t_{R 1}\right)=\frac{R_{23}\left(t_{R 1}\right)}{R_{23}\left(t_{0}\right)} \approx e^{-2 \delta V_{\tau 32}^{2} I_{\tau}}
$$

where

$$
\delta V_{\tau 32}^{2}=V_{\tau 3}^{2}-V_{\tau 2}^{2}
$$


which can be either positive, negative or zero. For the positive value, $\delta V_{\tau 32}^{2}>0$ as in hierarchical case[2], one gets the condition,

$$
R_{23}\left(t_{0}\right) \geq R_{23}\left(t_{R 1}\right)
$$

which implies the increase in the neutrino mass ratio $m_{\nu 2} / m_{\nu 3}$ with the decrease in energies[2]. If we start with degenerate neutrinos, $m_{\nu 2}=m_{\nu 3}$ at the scale $M_{R 1}$, then we would get $m_{\nu 2}>m_{\nu 3}$ at scale $m_{t}$. This shows that nearly degenerate neutrinos are not stable under quantum corrections[3].

The above relations in Eqs.(16-18) for $a=2,3$, can be generalised for any pair of mass eigenvalues $a=i, j$. For inverted hierarchical case [2] with $m_{\nu 1}>m_{\nu 2}$, we may have $\delta V_{\tau 21}^{2}<0$ which leads to

$$
R_{12}\left(t_{0}\right) \leq R_{12}\left(t_{R 1}\right)
$$

where the neutrino mass ratio $m_{\nu 1} / m_{\nu 2}$ decrease with the decrease in energies[2]. The effect of the running $\mathrm{VEV}$ does not change the textures of neutrino mass matrix and hence the MNS mixing matrix.

Next we turn to numerical analysis of the RGEs in the bottom-up approach in running from low energy scale $t_{0}$ to high energy scale, replacing $t_{R 1}$ by running $\mathrm{t}$ in the above equations (9-19). We make use of the following input values of the running fermion masses $m_{i}\left(m_{i}\right)$ of the third family:

$$
m_{t, b, \tau}=(166.5,4.2,1.785) G e V
$$

where, for heavy flavours(top and bottom quarks) the values are derived from input pole-masses $m_{t}^{\text {pole }}=175.6 \mathrm{GeV}[11]$ and $m_{b}^{\text {pole }}=4.7 \mathrm{GeV}[12,13]$ using two-loop RGEs in QCD. The initial input values for the top, the bottom and $\tau$-lepton Yukawa couplings at top-quark mass scale $t_{0}=\ln m_{t}$ in the RGEs in MSSM, are usually obtained as

$$
h_{t}\left(t_{0}\right)=m_{t} /(174 \sin \beta), h_{b, \tau}\left(t_{0}\right)=m_{b, \tau} /\left(174 \eta_{b, \tau} \cos \beta\right) .
$$


Using the CERN-LEP measurements at $M_{Z}=91.18 \mathrm{GeV}$,

$$
\alpha_{3}\left(M_{Z}\right)=0.118 \pm 0.004, \alpha^{-1}\left(M_{Z}\right)=127.9 \pm 0.1, \sin ^{2} \theta_{\omega}\left(M_{Z}\right)=0.2313 \pm 0.0003,(22)
$$

we obtain the values of gauge couplings at scale $t_{0}$ using one-loop RGEs, assuming the existence of one-light Higgs doublet $(n=1)$ and five quark flavours below $m_{t}$ scale,

$$
\alpha_{1,2,3}^{-1}\left(t_{0}\right)=58.42,29.67,8.89
$$

The QCD-QED rescaling factors [6] are calculated as

$$
\eta_{f}=(1.54,1.017), f=b, \tau
$$

As a result of the numerical analysis of the RGEs for Yukawa and gauge couplings at two-loop level[6] in the energy range $t_{0}<t<t_{U}$, the unification of three gauge couplings is observed at $M_{U}=1.82 \times 10^{16} \mathrm{GeV}$. The values of Yukawa couplings $\left(h_{t}, h_{b}, h_{\tau}\right)$, gauge couplings and values of integrals $I_{i}$ defined in Eqs. $(10,11)$ for different values of $\tan \beta=1.42-60.0$ are estimated at different energy scales.

We present our numerical results in Figs.(1-4) where the solid line refers to the analysis with the effects of running VEV in the present calculation (referred to as case A). We also present the corresponding results without the effect of running VEV in dotted line (referred to as case B) for comparison only. With a typical input value $V_{\tau 3}=\frac{1}{\sqrt{2}}$, the variation of the ratio $R_{3}(t)$ defined in Eq.(13), with energy scales $\mathrm{t}$ for two representative values of $\tan \beta=1.63$ and 57.29, are presented in Figs.1 and 2 respectively. These figures show the evolutions of neutrino mass eigenvalue $m_{\nu 3}$ with the increase in energy scale.

We observe that for high value of $\tan \beta=57.29$ in Fig.2, the evolution of the ratio $R_{3}(t)=m_{\nu 3}(t) / m_{\nu 3}\left(t_{0}\right)$ is almost stable in both cases A and B. However, for low value of $\tan \beta=1.63$ in Fig.1, there is a significant increase in $R_{3}(t)$ at higher energies in case B. For example, at $\mu=1.82 \times 10^{16} \mathrm{GeV}$, the ratio $R_{3}(t)$ is about 5.63 in case $\mathrm{B}$ 
as shown in Fig.1 by dotted line. Such unwanted feature which may cause instability, is not present in case A (solid line in Fig.1). Fig.3 shows the variation of neutrino mass, $R_{3}\left(t_{R 1}\right)=m_{\nu 3}\left(t_{R 1}\right) / m_{\nu 3}\left(t_{0}\right)$ at a particular scale, $t_{R 1}=27.63$ corresponding to $M_{R 1}=10^{12} G e V$, with different values of $\tan \beta=1.42-60$. We see that at low $\tan \beta \geq 1.42$ region there is a significant enhancement in $R_{3} \leq 5.3$ in case $\mathrm{B}$ whereas the ratio is stable in case $\mathrm{A}$ for all values of $\tan \beta$. For higher values of $\tan \beta$ the ratio is again stable in case B. The same analysis is true for the cases of other two mass eigenvalues with $a=1,2$. Similar analysis can be done for the evolution of $\triangle m_{i j}^{2}$ in Eq.(15), which would be very unstable in the low $\tan \beta$ region in case B. However, it is now stable for all values of $\tan \beta$ under radiative corrections at higher energies in case A.

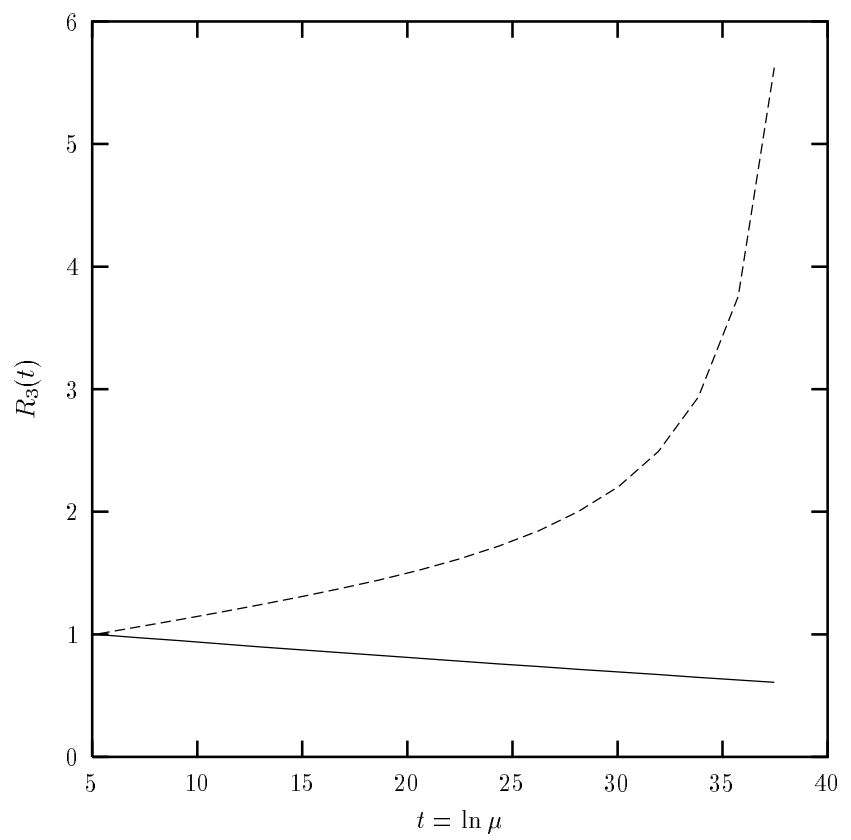

Fig.1 Variation of $R_{3}(t)=m_{\nu 3}(t) / m_{\nu 3}\left(t_{0}\right)$ with energies $t=\ln \mu$ for small value of $\tan \beta=1.63$. The results with and without the effect of running VEV, are shown in solid line and dotted line respectively. 


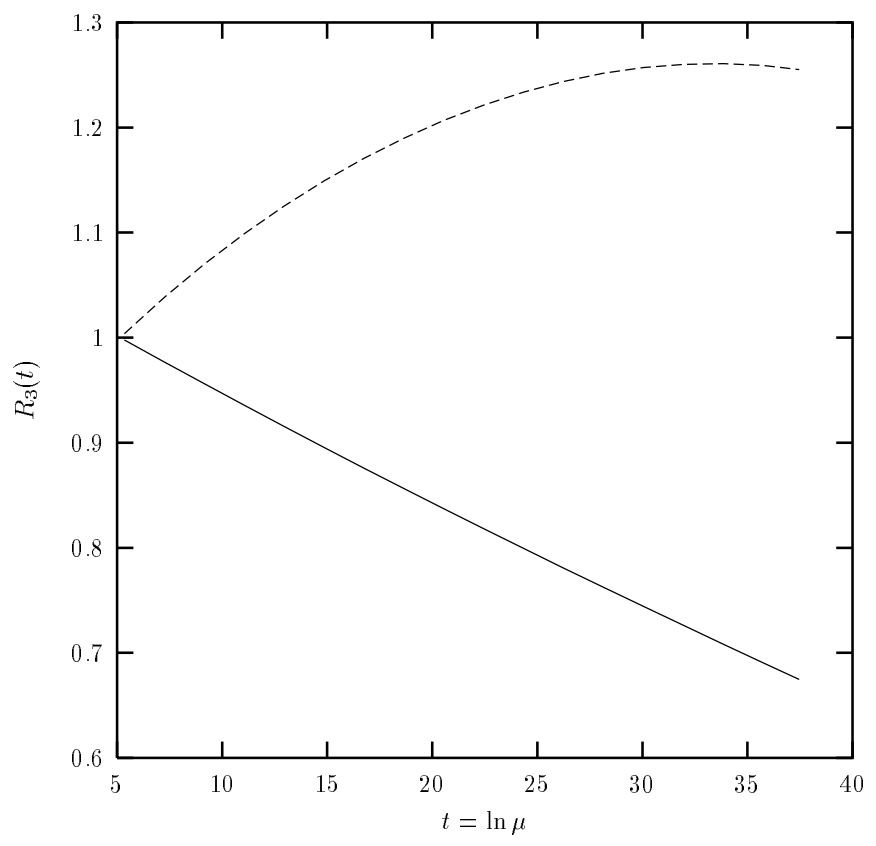

Fig.2 Variation of $R_{3}(t)=m_{\nu 3}(t) / m_{\nu 3}\left(t_{0}\right)$ with energies $t=\ln \mu$ for large value of $\tan \beta=57.29$. The results with and without the effect of running VEV are shown in solid line and dotted line respectively.

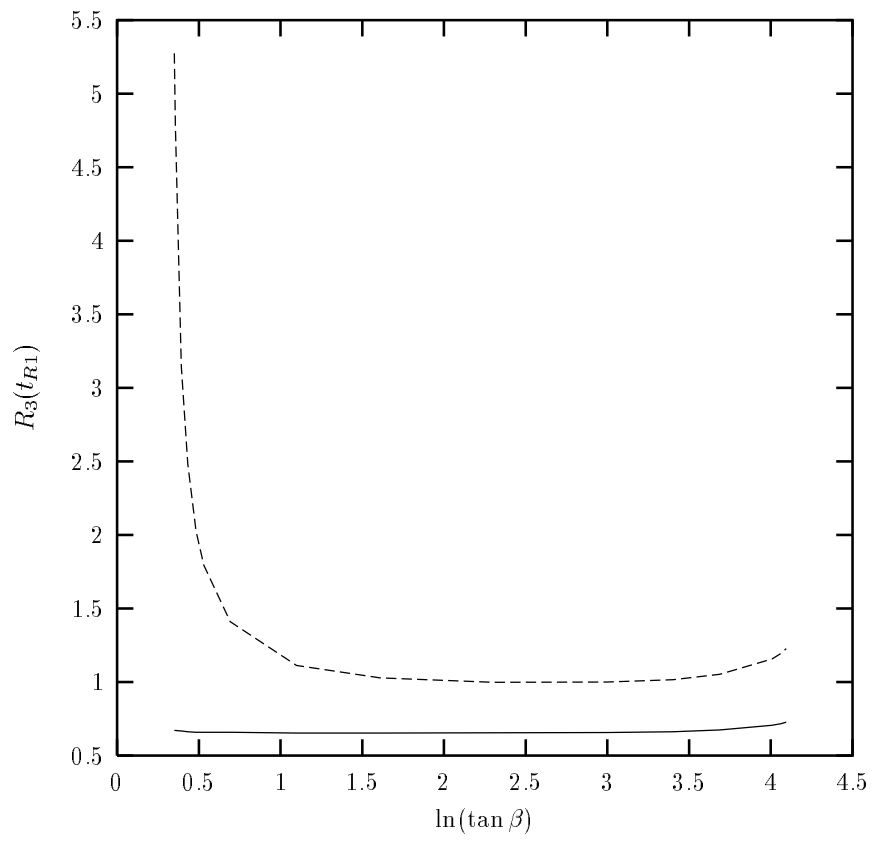

Fig.3 Variation of $R_{3}\left(t_{R 1}\right)=m_{\nu 3}\left(t_{R 1}\right) / m_{\nu 3}\left(t_{0}\right)$ with $\ln (\tan \beta)$ for $M_{R 1}=10^{12} \mathrm{GeV}$. The results with and without the effect of running VEV are shown in solid line and dotted line respectively. 


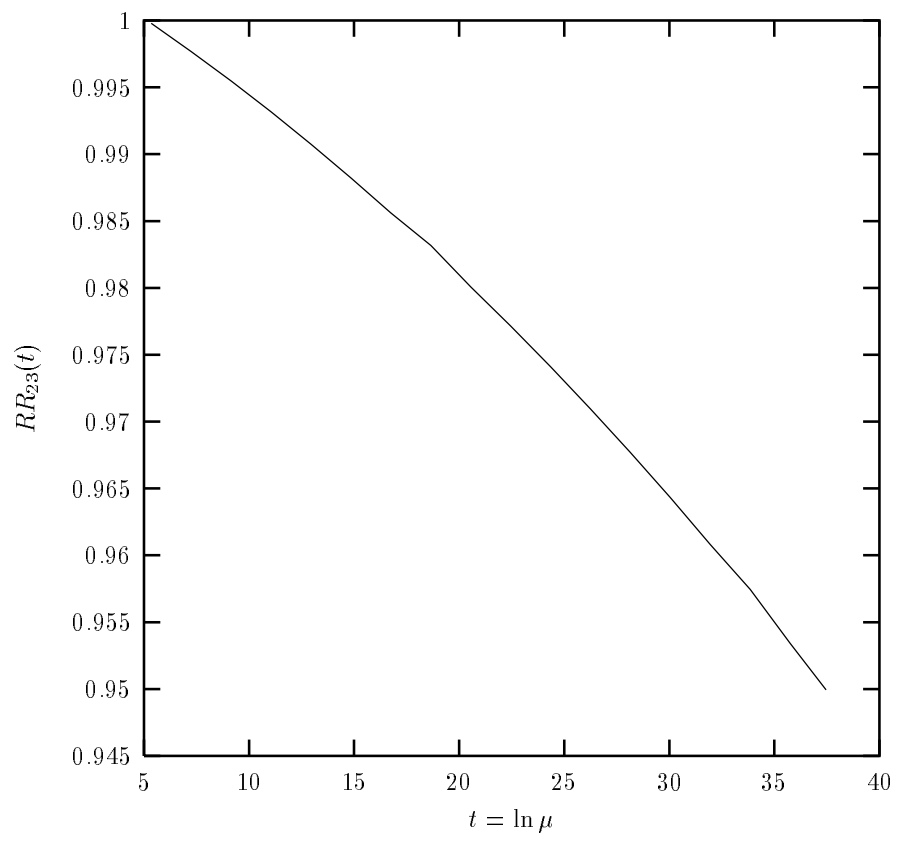

Fig.4 Variation of the ratio of the neutrino mass ratio $R R_{23}(t)=\left(\frac{m_{\nu 2}(t)}{m_{\nu 3}(t)}\right) /\left(\frac{m_{\nu 2}\left(t_{0}\right)}{m_{\nu 3}\left(t_{0}\right)}\right)$ with energies $t=\ln \mu$ for large value of $\tan \beta=57.29$.

Finally, we study the relative rates of the evolution of two neutrino mass eigenvalues in terms of their ratio, $R_{23}=m_{\nu 2} / m_{\nu 3}$ given in Eq.(16), in going from low to high energies. We consider high value of $\tan \beta=57.29$ where the effect of $I_{\tau}$ is large, and this ratio increases with the decrease in energies by a few percent only. This is shown in Fig. 4 where we present the evolution of the ratio of the ratios $R R_{23}(t)$ in Eq.(16) with energies. This leads to a mild increase in the hierarchical relation, $m_{\nu 2} / m_{\nu 3}$ at lower energies. As noted earlier, such hierarchical ratios are independent of the effect of running VEV. Finally we point out the changes aring from the running of VEV in the earlier calculations[2] of neutrino masses. The earlier results at low scale $m_{t}$ in Ref.[2] do not change at all. However if we prefer to express the neutrino mass matrix at higher scale $M_{R 1}$, then we have to take the effect of running VEV, $v_{u}\left(t_{R 1}\right)$ in place of $v_{u}\left(t_{0}\right)$, which modifies the earlier numerical results at the scale $M_{R 1}$.

To conclude, we have considered the contributions of scale-dependent vacuum 
expectation values (VEVs) of Higgs scalars in deriving one-loop analytic expression for running the left-handed Majorana neutrino masses with energies in the MSSM. This gives significant changes in the expression of the evolution of neutrino masses, and also increases the stability of the neutrino masses under quantum corrections even for low $\tan \beta$. We observed a mild decreasing trend of neutrino masses with higher energies, which is now common to that of all other fermion masses in nature.

\section{Acknowledgement}

The author would like to thank S.F.King for useful discussions and M.Oliveira for helping in computations.

\section{References}

[1] R.N.Mohapatra, hep-ph/9910365; H.Fritzsch and Z.Z.Xing, hep-ph/ 9912358; S.M.Barr and Ilja Dorsner, hep-ph/ 0003058; G. Altarelli, F. Feruglio and I. Masina, Phys. Lett. B472 (2000) 472; S.F.King, Nucl. Phys. B562 (1999) 57; ibid, Nucl. Phys.B576 (2000) 85; J. Ellis, G. K. Leontaris, S.Lola, D.V. Nanopoulos, Eur. Phys. J.C9 (1999) 389; S. Lola and G. Ross, Nucl. Phys.B553 (1999) 81; R. Barbieri, P.Creminelli and A. Romanino, Nucl. Phys. B559 (1999) 17.

[2] S.F.King and N.Nimai Singh,hep-ph/0007243, hep-ph/0006229.

[3] N.Haba and N.Okamura, Eur. Phys. J. C14 (2000) 347; J.A.Casas, J.R.Espinosa, A.Ibarra and I.Navarro, Nucl. Phys. B556 (1999) 3; hep-ph/9904395; hepph/9905381; J.Ellis, S.Lola, Phys. Lett. B458 (1999) 310; K.R.S.Balaji, A.S.Dighe, R.N.Mohapatra, M.K.Parida, Phys. Rev. Lett. 84 (2000) 5034;

[4] P.H.Chankowski, W.Krolikowski and S.Pokorski, Phys. Lett. B473 (2000) 109. 
[5] K.S.Babu, C.N.Leung and J.Pantaleone, Phys. Lett. B319 (1993) 319; P.H.Chankowski and Z.Pluciennik, Phys. Lett. B316 (1993) 312.

[6] V.Barger, M.S.Berger and P.Ohmann, Phys. Rev. D47 (1993) 1093; M.K.Parida and N.Nimai Singh, Phys. Rev. D59 (1999) 32002.

[7] M.Gell-Mann, P.Ramond and R.Slansky in Sanibel Talk, CALT-68-709, Feb 1979, and in Supergravity ( North Holland, Amsterdam 1979); T. Yanagida in Proc. of the workshop on Unified Theory and Baryon Number of the Universe, KEK, Japan, 1979; R. N. Mohapatra and G. Senjanovic, Phys. Rev. Lett. 44 (1980) 912; Phys. Rev. D23 (1981) 165.

[8] M.K.Parida and B.Purkayastha, Eur. J. Phys. C14 (2000) 159.

[9] H. Fusaoka and Y.Koide, Phys. Rev. D57 (1998) 3986; S.R.Juarez W., S. F. Herrera H., P. Kielanowski and G. Mora H., hep-ph/ 0009148.

[10] H.Arason, D.T.Castano, B.Kesthelyi, S.Mikaelian, E.J.Piard and P.Ramond, Phys. Rev. D9 (1992) 3945; D.J.Castano, E.J.Piard and P.Ramond, Phys. Rev. D49 (1994) 4882.

[11] M.C.Smith and S.S.Willenbrock, Phys. Lett. 79 (1997) 3825.

[12] N.Gray, D.Broadhurst, W.Grafe and K.Schilcher, Z. Phys. C48 (1990) 673.

[13] P. Abreu et.al., DELPHI Collab. CERN - PPE/97-141(1997); N. Nimai Singh and S. Biramani Singh, Indian J.Phys. 73A (3)(1999) 439. 